\title{
Collaborative Study for the Quantification of Total Contents of 2- and 3-Monochloropropanediol and Glycidol in Food Emulsifiers by GC-MS
}

\author{
Jan Kuhlmann ${ }^{1}$ \\ ${ }^{1}$ SGS Germany GmbH
}

June 29, 2021

\begin{abstract}
An international robin round was carried out to validate a method for the quantification of 2-monochloropropane-1,3-diol (2MCPD), 3-monochloropropane-1,2-diol (3-MCPD) and 2,3-epoxy-1-propanol (Glycidol) being present as fatty acid esters in plant-based food emulsifiers. The evaluated method was a modification of the American Oil Chemist's Society (AOCS) Official Method Cd29b-13. Briefly, this method consists from parallel analysis of two sample aliquots that are spiked with different sets of internal standards. Mild alkaline interesterification overnight in the freezer releases the core analytes. Reaction stop and glycidol conversion into monobromopropanediol (MBPD) is realised by addition of acidified sodium bromide solution. Subsequently, matrix removal and analyte extraction are achieved by two liquid/liquid (l/l) extraction steps. After derivatisation with phenylboronic acid (PBA) the final extracts are analysed by gas chromatography-mass spectrometry (GC-MS). Quantification is carried out by internal one-point-calibration. 6 laboratories from 4 European countries participated in the trial and reported 8 data sets for 10 test materials (mono- and diacylglycerides as well as polyglycerol polyricinoleates) that were analysed as blind duplicates, giving a total of 20 samples. Result outliers were eliminated according to accepted standards. At 2-MCPD levels above $0.02 \mathrm{mg} / \mathrm{kg}$, 3-MCPD levels above $0.06 \mathrm{mg} / \mathrm{kg}$ and glycidol levels above $0.22 \mathrm{mg} / \mathrm{kg}$ repeatability (RSDr) ranged from $1.5 \%$ to $24.9 \%$, reproducibility (RSDR) ranged from $7.8 \%$ to $29.0 \%$ and HORRATR-values ranged from 0.5 to 1.7 . The tested method showed to be suitable for the determination of 2-MCPD, 3-MCPD and glycidol in food emulsifiers consisting from mono- and diacylglycerides as well as polyglycerol polyricinoleates.
\end{abstract}

\section{Collaborative Study for the Quantification of Total Contents of 2- and 3- Monochloropropanediol and Glycidol in Food Emulsifiers by GC-MS}

Jan Kuhlmann

Jan Kuhlmann, SGS Germany GmbH, Weidenbaumsweg 137, 21039 Hamburg, Germany

Abstract An international robin round was carried out to validate a method for the quantification of 2monochloropropane-1,3-diol (2-MCPD), 3-monochloropropane-1,2-diol (3-MCPD) and 2,3-epoxy-1-propanol (Glycidol) being present as fatty acid esters in plant-based food emulsifiers. The evaluated method was a modification of the American Oil Chemist's Society (AOCS) Official Method Cd29b-13. Briefly, this method consists from parallel analysis of two sample aliquots that are spiked with different sets of internal standards. Mild alkaline interesterification overnight in the freezer releases the core analytes. Reaction stop and glycidol conversion into monobromopropanediol (MBPD) is realised by addition of acidified sodium bromide solution. Subsequently, matrix removal and analyte extraction are achieved by two liquid/liquid (l/l) extraction steps. After derivatisation with phenylboronic acid (PBA) the final extracts are analysed by gas chromatographymass spectrometry (GC-MS). Quantification is carried out by internal one-point-calibration. 6 laboratories from 4 European countries participated in the trial and reported 8 data sets for 10 test materials (mono- 
and diacylglycerides as well as polyglycerol polyricinoleates) that were analysed as blind duplicates, giving a total of 20 samples. Result outliers were eliminated according to accepted standards. At 2-MCPD levels above $0.02 \mathrm{mg} / \mathrm{kg}, 3$-MCPD levels above $0.06 \mathrm{mg} / \mathrm{kg}$ and glycidol levels above $0.22 \mathrm{mg} / \mathrm{kg}$ repeatability $\left(\mathrm{RSD}_{\mathrm{r}}\right)$ ranged from $1.5 \%$ to $24.9 \%$, reproducibility $\left(\mathrm{RSD}_{\mathrm{R}}\right)$ ranged from $7.8 \%$ to $29.0 \%$ and $\mathrm{HORRAT}_{\mathrm{R}^{-}}$ values ranged from 0.5 to 1.7. The tested method showed to be suitable for the determination of 2-MCPD, 3 -MCPD and glycidol in food emulsifiers consisting from mono- and diacylglycerides as well as polyglycerol polyricinoleates.

Keywords Collaborative study / 2-MCPD / 3-MCPD / glycidol / food emulsifiers

\section{Introduction}

3-MCPD is known to be a heat-induced contaminant that can be formed by different food processing techniques such as heating (e.g. smoking, roasting, baking, grilling) of various kinds of foods, (Hamlet, 2002). 2- and 3-monochloropropanediol fatty acid esters (2- and 3-MCPDE) as well as glycidyl fatty acid esters (GE) are also heat-induced food contaminants that are ubiquitously found in refined edible oils/fats, (Zelinkova, 2006; Weisshaar, 2010; Kuhlmann, 2011). 3-MCPD has been classified by the International Agency for Research on Cancer (IARC) as possibly carcinogenic to humans (category 2B) (International Agency for Research on Cancer, 2012) while glycidol has shown genotoxic properties and was ranked as probably carcinogenic to humans (category 2A), (International Agency for Research on Cancer, 2000). Animal experiments as well as human studies have shown that digestion of 2-MCPDE, 3-MCPDE and GE releases the core compounds completely or to large extent, (Abraham, 2013; Appel, 2013; Abraham 2021). Correspondingly, 3-MCPD, glycidol and their fatty acid esters are considered to have the potential to show adverse health effects when ingested. The European Food Safety Authority considered that in terms of exposure the fatty acid bound compounds do show the same toxicity as the free analytes on molar base, (EFSA CONTAM panel, 2016).

It appears evident that food emulsifiers might contain MCPDE as well as GE if they are directly or indirectly derived from edible oils/fats when heat treatment is applied as deodorisation or distillation during manufacturing. Since 2018, the European Food Safety Authority (EFSA) and the European Commission (EC) released calls for technical and toxicological data on different classes of emulsifiers used as food additives, (EFSA, 2018; EC, 2018a; EC, 2018b). These calls for data were launched as a follow-up to EFSA's re-evaluation of a number of food emulsifiers, (EFSA, 2017a; EFSA, 2017b; EFSA, 2017c), in line with EU Regulation 257/2010, (EU 2010). By these calls, analytical data and/or the lowest technologically achievable levels of 3-MCPD/3-MCPD esters/glycidol/glycidyl esters were requested.

From a regulatory perspective, EU Regulation 2020/1322, amending Regulation 1881/2006, sets a maximum level of $1000 \mu \mathrm{g} / \mathrm{kg}$ for glycidol in the form of glycidyl fatty acid esters in vegetable oils/fats placed on the market for the final consumer, or for use as a food ingredient. In oils/fats used for the production of baby food and cereal-based foods for infants and young children, the maximum level for GE is $500 \mu \mathrm{g} / \mathrm{kg}$. With the same regulation, maximum quantities for the sum of free and fatty acid bound 3-MCPD (analyte group) are set for the same foods for which maximum levels for GE have been established. Due to the lower 3-MCPD formation potentials, a stricter maximum level $(1250 \mu \mathrm{g} / \mathrm{kg})$ for the sum of 3-MCPD and 3-MCPD fatty acid esters, expressed as $3-M C P D$ is set for oils and fats from coconut, maize, rapeseed, sunflower, soybean, palm kernel and olive oils and mixtures of oils and fats with oils and fats only from this category. A higher maximum level of $2500 \mu \mathrm{g} / \mathrm{kg}$ applies to other vegetable oils (including olive pomace oils), fish oils and oils from other marine organism and mixtures of oils and fats with oils and fats only from this category. For oil mixes from both categories with quantitatively known ingredients, the category-related maximum levels apply to the single oil or fat components of the mix. In case of oil and fat mixes of unknown composition, the higher maximum level of $2500 \mu \mathrm{g} / \mathrm{kg}$ applies. For vegetable oils and fats, fish oils and other marine oils that are destined for the production of baby food and processed cereal-based food for infants and young children the maximum level for the sum of 3-MCPD and 3-MCPD fatty acid esters, expressed as 3-MCPD is set to $750 \mu \mathrm{g} / \mathrm{kg}$. Finally, for infant formula, follow-on formula and foods for special medicinal purposes for infants and young children as powders the maximum level is addressed as $125 \mu \mathrm{g} / \mathrm{kg}$ for powders and as 15 $\mu \mathrm{g} / \mathrm{kg}$ for liquids, (EU, 2020). This Regulation does not apply directly to food emulsifiers. However, there 
remains a correlation as food emulsifiers might be used for the production of certain types of baby and young children foods. Furthermore, from practical perspective, it can be assumed that food control laboratories will analyse the lipid phase extracted from compound foods in order to conclude, if the oils used for the production of the corresponding foods match official regulation or other limits set by producers or retailers. This approach might give improper results in case that food emulsifiers contributed to the 3-MCPD and glycidol levels of the extracted lipid fraction. Considering these circumstances, the availability of a reliable analytical method for the determination of 3-MCPD, glycidol and their derivatives in food emulsifiers seems to be of high relevance.

Several officially validated analytical methods are available for the parallel determination of ester-bound 3 -MCPD and ester-bound glycidol in edible oils \& fats. The most common methods used seem to be the AOCS Official Methods Cd 29a-13, Cd29b-13 and Cd29c-13 (AOCS, 2017a-c) which also were adopted as ISO standards 18363-3, 18363-2 and 18363- 1 (ISO, 2015; ISO, 2018; ISO, 2017). AOCS Cd 29c-13 is also equal to the standard C-VI 18 (10) of the German Society for Fat Research (DGF), (DGF, 2011). The AOCS Cd29 / ISO 18363 / DGF C-VI approaches are referred to as indirect methods that are based on chemical transesterification so as not to determine the various single fatty acid ester derivatives of 3-MCPD and glycidol but to release these core components in their free form. The resulting highly reactive free glycidol is stabilised by transformation into monobromopropanediol (AOCS Official Methods Cd 29a, b-13) or into induced 3-MCPD (AOCS Official Method Cd29c-13, Part A). At present, food emulsifiers are not within the scope of these methods. However, while the determination of (2-)3-MCPD or (2-)3-MCPDE does not include a basic chemical conversion of the molecular core structures, there does not seem to be any apparent theoretical indication that the validated methods cannot be used for neutral food emulsifiers in general. Nevertheless, there is no practical confirmation of this assumption so far and it might remain questionable if the limits of quantification are the same as with oils and fats. The determination of GE is even more critical as it has been reported that using the AOCS Official Method Cd 29a-13 for mono- and diacylglycerides can lead to GE overestimations, (Zelinkova, 2017). Furthermore, unpublished communications of analytical laboratories and their customers suggest that the validated indirect methods may give unreliable GE results, such as false positives, overestimations or inconsistent values. In addition, internal research by the author has shown instances where using AOCS Official Method Cd 29b-13 to analyse food emulsifiers resulted in GE levels that increased or decreased over time while 3-MCPD levels remained constant (data not shown). In theory, these effects might be caused by GE decomposition during storage/sample preparation or from the occurrence of other unstable GE-simulating compounds. In particular, melting samples for aliquot preparation has been shown in individual cases to significantly accelerate GE decomposition (data not shown). Vice versa, artefact formation during sample preparation cannot be excluded as a possible source of GE overestimation or false-positive results. In order to overcome these drawbacks in the determination of GE in food emulsifiers, a new approach has been developed that is based on a modification of the AOCS Official Method Cd 29b-13. Beside minor modifications in terms of sample weight and solvents used, the main difference to the original method is the quantitative differentiation of MBPD isomers which seem to be formed in a relatively constant ratio when glycidol reacts with acidified sodium bromide solution. MBPD artefact formation through other compounds as glycidol seems to be related to the 3-MBPD isomer but not to the isomer carrying bromine in the 2-position. Correspondingly, the ratio of both isomers is determined and in case of increased ratio 3-MBPD : 2-MBPD the quantification of glycidol is carried out via 2-MBPD.

The reported study was undertaken to evaluate the applicability of this new analytical approach for the quantitative determination of 2- and 3-MCPD and their fatty acid esters as well as glycidyl fatty acid esters in food emulsifiers that are prone to contain these analytes and to provide validation data accordingly. 


\section{Materials and Methods}

\section{Collaborative Study}

On request of the European Food Emulsifier Manufacturer's Association (EFEMA), which initiated this study, participation was restricted to SGS Germany GmbH and EFEMA members. Some participants were new to the field of processing contaminants trace analysis, others had a high level of expertise. Every laboratory received ten food emulsifier test materials as blind duplicates, giving a total of 20 samples. A study protocol was supplied by e-mail and as a print-out to accompany the test samples. In addition to general instructions and advice on sample storage conditions, every protocol included the specific method to analyse 2- and 3-monochloropropanediol esters and glycidyl esters. The protocol also included a goods receipt form, an EXCEL-based raw data evaluation template and a result submission sheet. No training sample nor internal standard materials or solutions were supplied. Participants had to prepare internal standard stock and working solutions according to the method SOP.

All participants were asked to perform analyses on each of the 20 test materials strictly following the method protocol and to send back the results within four weeks of receiving the samples, together with any comments regarding observed difficulties or anomalies and differences of the applied procedure to the study protocol. However, due to laboratory limitations caused by the coronavirus pandemic starting in early 2020, a delay in result submission was accepted. For the same reason, results were included even if deviations to the study protocol were identified. This related, in particular, to the use of other chromatographic injections systems such as programmable temperature vaporisers (PTV). If ester hydrolysis rates were defined by the study protocol as potentially insufficient, non-repeated measurements were also accepted. Furthermore, it was decided not to insist on the use of isotope-labelled free D2-MCPD as an internal standard. No information was supplied for the specific identity of the test materials prior to the study. However, in individual cases, it could have been possible in theory to identify pairs of blind duplicates by their visual appearance. Participants were advised to contact the Study Director if they experienced any problem or had questions about the method or the protocol. The study protocol did not contain any specific recommendation to exclude results below the individual laboratories limits of quantification (LOQs) as the intention was to determine the method applicability range based on the statistical evaluation. Mean values were derived if multiple data sets were submitted. This is not in line with recommendations for official method validation, but it should reflect that some of the participating laboratories were new to this field of analysis. Results continued to be submitted between February and June 2020.

Six laboratories from four countries (3 x Denmark, 1 x France, 1 x Germany, 1 x Ireland) participated in this study and reported results. However, due to the statistical limitations that would result from such a small number of participants, one laboratory reported three data sets that were generated independently at different times (start, middle and end of the testing period) by different lab technicians using separate workplaces and separately prepared standard working solutions. Furthermore, measurements of the final sample extracts were carried out using three single GC-MS instruments and the raw data was evaluated by

another person so that the analysts carrying out sample preparation had no information on the quantitative results. The following laboratories participated in the ring trial:

DuPont Nutrition Biosciences ApS, Brabrand, Denmark

Dupont Nutrition \& Health, Grindsted, Denmark

Kerry EMEA Global Technology \& Innovation Centre, Kildare, Ireland

Oleon Innovation, Compiègne Cedex, France

Palsgaard A/S, Juelsminde, Denmark

SGS Germany GmbH, Hamburg, Germany 


\section{Sample scheme and preparation}

Prior to the trial, 40 food emulsifier samples supplied by EFEMA were tested for specific analyte levels, stability and homogeneity. For this purpose, all samples were analysed three to six times over a period of six to eight weeks, using the analytical method that would be used by the participants of the multi-laboratory trial. As this approach required the preparation of duplicate samples, 6 to 12 data sets were available per sample. First, the results were checked for trends of obviously increasing or decreasing analyte levels. No such trend was observed for 2- and 3-MCPD esters. However, individual samples indicated decreasing GE levels. Homogeneity was assumed to be satisfactory if the observed repeatability of analyte results did not significantly exceed the range the testing laboratory would usually observe for common oils and fats.

Out of the set of 40 food emulsifiers, ten test materials were selected to fulfil the following criteria:

1. In terms of possible MCPDE/GE occurrence and EFSA call for data, the test samples should represent the most important (sub-)classes of food emulsifiers.

2. The selected test materials should cover commonly occurring and high analyte contents and at levels that might become important under current and future regulation of 3-MCPD and GE in edible oils and fats. Furthermore, the study aimed to include samples with different ratios of GE:MCPDE.

3. Special emphasis was placed on including blank samples to check for possible GE artefact formation during sample preparation or measurement.

4. Sample homogeneity and analyte stability of the test samples should have been shown to be satisfactory such that no significant issues were assumed to occur for the duration of the trial. The acceptance criterion was set as a pre-testing RSDr value below $10 \%$ for all analytes present at a level [?] 0.1 $\mathrm{mg} / \mathrm{kg}$.

It was decided not to include spiked blank samples to determine trueness as a previous in-house validation of the method revealed satisfying results when analysing certified vegetable oils from previous proficiency tests. In addition, good recoveries were achieved when spiking food emulsifier blank samples. However, spiking food emulsifiers that contained glycidol, which showed significantly deviating GE results when comparing the nonmodified and the modified AOCS Official Method Cd 29b-13, resulted in satisfactory spiking recoveries for both methods. These unexpected findings revealed the infeasibility of GE spiking for the purpose of verifying method trueness in this case.

Table 1 presents the samples included in this method validation study.

Amongst the selected samples, the focus was set very much on the EU food additive E 471 due to the assumption that mono- and diacylglycerides (MAG and DAG) are most likely to contain relevant levels of MCPDE and GE. The intention was to include as many different subclasses of E 471 as possible. For this reason, half of the $\mathrm{E} 471$ test materials were distilled monoacylglycerides (DMG), the other half was mixtures of MAG and DAG (MDG). Furthermore, both groups contained emulsifiers derived from different classes of fatty acids, namely saturated, monounsaturated and polyunsaturated fatty acids.

Based on the pre-testing results, the "Ink" sample was chosen as an E 471 material that contains no analyte or only a minor quantity $(<0.1 \mathrm{mg} / \mathrm{kg})$. This choice was made to verify that the applied method does not carry the risk of significant analyte overestimations or false-positive results by artefact formation during sample preparation or measurement. "Scissors" represented DMG, containing low analyte amounts below the maximum levels that are set for oils and fats by recent EU regulation. With this sample, the glycidol content was expected to range below or at the limit of quantification (LOQ) of the method. Conversely, "Orange" served to reflect a DMG material with 3-MCPD and GE contents above regulatory limits. The test material "Green" exhibited a large excess of 3-MCPD in comparison to glycidol, while "Peach", in contrast, contained significantly more glycidol than 3-MCPD. The "Monday" MDG sample was expected to represent comparably low but quantifiable analyte levels below the regulatory limits while "March" contained glycidol as a major analyte not too far above the $1 \mathrm{mg} / \mathrm{kg}$ level and had a negligible MCPD content. 
The sample named "Olive Tree" contained minor amounts of MCPD and the highest glycidol level of the selected test specimens. This choice was made to obtain information on the analytical range of the method. Finally, the test materials "Caravaggio" and "Berlioz" served as single representatives of two classes of polyglycerol esters, namely E 475 and E 476. "Caravaggio" did not contain any of the target analytes in significant amounts. However, this sample showed positive but inconsistent GE findings when applying the non-modified quantification approach of the official method AOCS Cd 29b-13. Similar effects were observed with the test materials "Scissors", "Green" and "Monday". During pre-testing, none of the investigated E 476 polyglycerol polyricinoleate samples contained significant amounts of any of the target analytes. In this regard, "Caravaggio" and "Berlioz" served as blank samples to check that no false-positive findings could occur when using the tested method.

To prepare aliquots, samples that were powders, pellets, flakes or liquid were transferred directly into $200 \mathrm{~mL}$ screw cap plastic vessels that were labelled with the corresponding anonymous sample code. Test materials listed in Table 1 as "(solid)" were molten and transferred in liquid form. A minimum amount of $25 \mathrm{~g}$ was obtained for most of the sample aliquots. However, the quantities were lower in certain cases due to a limited availability of the corresponding original sample. Prior to sample filling the vessels were tested to ensure they were free of glycidol and MCPD. All samples were stored frozen $(-25 \operatorname{deg} \mathrm{C}+/-3 \operatorname{deg} \mathrm{C})$ prior to distribution to the participants. Dry ice was used as a cooling agent during transit.

\section{Principle of the test method}

For the determination of free and bound 2-MCPD, free and bound 3-MCPD and bound glycidol as free 2-MCPD, free 3-MCPD and free 3-MBPD respectively, two aliquots (A and B) of the sample are spiked with surrogate standards ( $\mathrm{D}_{5}-2-\mathrm{MCPD}, \mathrm{D}_{5}-3-\mathrm{MCPD}, \mathrm{D}_{5}$-glycidylester in assay $\mathrm{A}$ and $\mathrm{D}_{5}-2-\mathrm{MCPD}-1,3-$ diester, $\mathrm{D}_{\mathbf{5}}-3$-MCPD-1,2-diester in assay B) and dissolved in tertiary butyl methyl ether ( $t$ BME). Both assays are processed in parallel. The addition of a diluted solution of sodium hydroxide or sodium methoxide in methanol at low temperatures will release free 2-MCPD, free 3-MCPD and free glycidol over a period of $8 \mathrm{~h}$ to $16 \mathrm{~h}$. This reaction is stopped by adding an excess of sodium bromide in acidic solution. Under acidic conditions, free glycidol reacts with inorganic bromide to form 3-MBPD and 2-MBPD. Undesired non-polar compounds in the sample are removed by multiple extraction of the aqueous phase with $t$ BME and iso -hexane. The analytes, together with the surrogate standards, are transferred into an organic phase by multiple extraction of the aqueous phase with diethyl ether, ethyl acetate or a mixture of both solvents. Derivatisation of the diol analytes to form dioxaborolane derivatives is carried out in the organic phase by reaction with phenylboronic acid (PBA). In order to remove excess amounts of PBA, the sample extract is subsequently placed over a small amount of anhydrous sodium sulfate and evaporated to dryness under a stream of nitrogen before the soluble residue is finally resorbed iniso -octane and measured by GC/MS, applying separate thermal ramping of the injector and the GC column.

The alkali-catalysed transesterification at low temperature minimises the undesired transformation of 3MCPD into glycidol that occurs to a significant extent at room temperature. Nevertheless, in the event that large amounts of 3-MCPD are present, even a minor transformation into glycidol could artificially increase the glycidol results from assay A. In order to achieve the correct glycidol results, assay B is used to quantify the undesired 3-MCPD-glycidol transformation by determining the amount of $\mathrm{D}_{5}$-glycidol generated from $\mathrm{D}_{5}$-3-MCPD-diester in the sample preparation. The corresponding transformation ratio is used to correct the glycidol value derived from assay A. 3-MCPD has shown to be converted slightly faster via glycidol into 3-MBPD than $\mathrm{D}_{5}-3$-MCPD via $\mathrm{D}_{\mathbf{5}}$-glycidol into $\mathrm{D}_{\mathbf{5}}-3$-MBPD. Consequently, an isotopic factor has to be determined empirically and considered for the quantitative determination of the amount of glycidol that has been generated accidentally from the non-labelled 3-MCPD by alkaline treatment in assay A.

Quantification of the analytes is carried out by internal one-point calibration using the corresponding $\mathrm{D}_{\mathbf{5}}$ -esters as surrogate standards. Peak integration is carried out for corresponding mass-to-charge ratios. Therefore, no external calibration is necessary. Likewise, no analyte recoveries have to be considered. However, the cleavage rates of MCPD mono- and diesters might be different and as only $\mathrm{D}_{\mathbf{5}}$-MCPD-diesters serve 
as internal standards, ester cleavage should have proceeded on a large scale. Therefore, the degree of ester cleavage is monitored by calculating the differences in 3-MCPD results between assay A and B. In order to avoid glycidol overestimations due to artefact formation from bromide with reactive compounds that might be present in food emulsifiers, quantification of glycidol via 2-MBPD is carried out for control and correction purposes. The essential step in glycidol quantification by this method is to exclude values resulting either from 3-MBPD or from 2-MBPD determination when one of the two results is greater than $10 \%$ of the mean value of both results. The exact procedure as provided for the trial is given as Annex A . A few editorial changes to the original protocol are included as a result of participant input during the trial.

\section{Statistical Evaluation}

In order to include as much data in the statistical evaluation as possible, it was decided not to exclude results that might have been classified as being below the individual laboratories limit of quantification. Instead, the methodological limits of quantification were derived as method applicability levels by statistical analysis of the submitted data.

Data sets were evaluated statistically following ISO 5725-2:1994 as far as possible, (ISO, 1994). In this regard, numerical outlier testing was performed by first applying Cochran's test and then Grubbs's test. Cochran's test helps to identify outliers and stragglers in terms of repeatability. It identifies a significant deviation in duplicate sample results from the average distribution of duplicate sample results. To determine outliers and stragglers in terms of reproducibility, the highest and lowest values were checked as single results as well as result couples by using Grubbs's test. Statistical evaluation included the determination of mean values, absolute and relative repeatability and reproducibility, reproducibility limits and HorRat values, (Horwitz, 2006). Method applicability for the quantitative determination of ester-bound glycidol and free and esterbound 2- \& 3-MCPD was confirmed to ensure that for a particular combination of analyte level and sample type the corresponding relative repeatability $\left(\mathrm{RSD}_{\mathrm{R}}\right)$ did not exceed $30 \%$ and the corresponding HorRat R-value was within the normal range 0.5 [?] HorRat R [?] 1.5 or in a higher but acceptable range $1.5<$ HorRat R [?] 2. In general, the exclusion of one outlier or straggler within the dataset $(n=8)$ was accepted. If two out of eight results had to be excluded, method applicability was confirmed only if the corresponding HorRat $\mathrm{R}$ value was found to be in the normal range 0.5 [?] HorRat R [?] 1.5. Using a similar procedure, HorRat $\mathrm{r}$ values were derived and evaluated for repeatability. The corresponding data is not shown as no results were excluded purely due to insufficient reproducibility.

\section{Results and Discussion}

Tables 2.1 - 2.3 display summaries of the statistical evaluation. The laboratory order corresponds to the time of result submission.

In general, the applied method showed a satisfactory applicability for the determination of glycidyl fatty acid esters and the sum of 3-MCPD and 3-MCPD fatty acid esters in all tested food emulsifiers. Only the samples that contained no analyte or non-quantifiable minor analyte contents as evidenced during pre-testing caused an inacceptable number of results to be excluded or the reproducibility relative standard deviation to exceed the accepted maximum value of $30 \%$ and/or the corresponding HorRat $\mathrm{R}$ value to be larger than 2. These findings were expected in terms of blank or semi-blank samples and might be considered to be an indication that the method is generally unlikely to generate false-positive results. This applies to the results for glycidol ${ }^{1)}$ in the samples "Ink" and "Scissors", to 2- \& 3-MCPD ${ }^{2)}$ in sample "March" and to all analytes in both polyglycerol samples "Caravaggio" and "Berlioz". Considering that a data set of eighty individual results was available for every analyte, the number of excluded outliers or stragglers differed. Twelve glycidol results were excluded, eight 3-MCPD results and four 2-MCPD results respectively. Furthermore, the results indicate that method applicability was achieved for much lower 2- and 3-MCPD contents in comparison to glycidol where the LOQ was estimated to range between $0.10 \mathrm{mg} / \mathrm{kg}$ and $0.20 \mathrm{mg} / \mathrm{kg}$. These differences seem to be understandable for several reasons. Amongst the tested analytes, glycidol is the most unstable 
one and, using the tested method, it undergoes a chemical reaction that changes its core structure. The corresponding chemical reaction of epoxy-ring opening and MBPD formation is certainly a source of error as it might be influenced by the $\mathrm{pH}$ value, other nucleophiles or the possible presence of other reactive components. Moreover, glycidol reacts to produce the two isomers 2-MBPD and 3-MBPD, which are both considered for quantitation. Thus, the signal intensities are lowered correspondingly which results in greater measurement uncertainty. A further impact on repeatability and reproducibility of the glycidol determination can be assumed from the undesired conversion of 3-MCPD into induced glycidol. This effect is measured and corrected within the tested method, but these steps can also be considered with evidence to expand the result variation.

\section{Results for glycidol}

At method applicability levels between $0.22 \mathrm{mg} / \mathrm{kg}$ and $10.35 \mathrm{mg} / \mathrm{kg}$, the HorRat values for glycidol ranged between 0.7 and 1.4, showing good correlation to the expected normal range of $1+/-0.5$. The corresponding reproducibility relative standard deviations $\left(\mathrm{RSD}_{\mathrm{R}}\right)$ showed values from $10.1 \%$ to $29.0 \%$ while the relative repeatability $\left(\mathrm{RSD}_{\mathrm{r}}\right)$ ranged from $3.9 \%$ to $24.9 \%$ and did not exceed the acceptable maximum level of 30 \%. As expected, the $\mathrm{RSD}_{\mathrm{R}}$ and $\mathrm{RSD}_{\mathrm{r}}$ values increase in general with decreasing analyte concentrations. The HorRat value was only higher than expected at 1.7 for "Olive Tree", which contained $28.20 \mathrm{mg} / \mathrm{kg}$ glycidol, the highest level among the test materials. Nevertheless, it was still in the acceptable range < 2. At the same time, the corresponding $\mathrm{RSD}_{\mathrm{R}}$ value of $16.1 \%$ was not close to the critical limit of $30 \%$. The reason for the HorRat value being higher than commonly found for this concentration range, might be that the analyte level exceeded the default one-point calibration maximum level of $5 \mathrm{mg} / \mathrm{kg}$ almost by a factor of six. In addition, one lab submitted results that were roughly $30 \%$ lower than the average for all mean glycidol contents $>1 \mathrm{mg} / \mathrm{kg}$. This deviation was not big enough to become an outlier, but it had a relatively high impact on the method reproducibility due to the small number of participants. As the repeatability relative standard deviation for glycidol in the "Olive Tree" sample was quite low as expected $\left(\mathrm{RSD}_{\mathrm{r}}=1.9 \%\right)$, this indicates that the relatively high deviation in results between the participants might be caused by variations in the concentrations of the calibration standards used. The second impact on the reproducibility of glycidol-determination is given by the relative amount of 3-MCPD present in parallel with glycidol. As pointed out in the previous paragraph, a high ratio of 3-MCPD : glycidol should negatively impact the precision of the glycidol determination. This effect becomes obvious when comparing the results of the two samples "Green" and "Monday", which both showed almost the same amount of glycidol. "Green" contained 3-MCPD in a more than eightfold excess and consequently, repeatability and reproducibility of the glycidol results were close to the exclusion limits $\left(\mathrm{RSD}_{\mathrm{r}}=24.9 \% ; \mathrm{RSD}_{\mathrm{R}}=29.0 \%\right)$. In comparison, "Monday", which contained a 2.7-fold excess of 3-MCPD compared with glycidol, had more satisfactory $\mathrm{RSDr}$ and $\mathrm{RSD}_{\mathrm{R}}$ values for glycidol $\left(\mathrm{RSD}_{\mathrm{r}}=4.8 \% ; \mathrm{RSD}_{\mathrm{R}}=19.3 \%\right)$. The best reproducibility for glycidol results $\left(\mathrm{RSD}_{\mathrm{R}}=10.1 \%\right.$ ) was found for "March", which had an elevated glycidol level of $1.4 \mathrm{mg} / \mathrm{kg}$ and contained no significant amounts of 3-MCPD. A data summary is displayed in Table 3 .

\section{Results for 3- and 2-MCPD}

For 3-MCPD method applicability levels above $0.06 \mathrm{mg} / \mathrm{kg}$ and for 2-MCPD concentrations above 0.02 $\mathrm{mg} / \mathrm{kg}$, all data showed to be valid with no $\mathrm{RSD}_{\mathrm{R}}$ value exceeding $30 \%$ or any HorRat $\mathrm{R}$ value $>2$. The HorRat $\mathrm{R}$ values varied from 0.5 to 1 within a range of $0.06 \mathrm{mg} / \mathrm{kg}$ to $2.86 \mathrm{mg} / \mathrm{kg}$ (3-MCPD) and from 0.5 to 0.8 within a range $0.02 \mathrm{mg} / \mathrm{kg}$ to $0.72 \mathrm{mg} / \mathrm{kg}(2-\mathrm{MCPD})$. These values are slightly better than expected but they are not in the range $<0.5$ which could be classified as being questionably good. This might be due to the results being averaged, but also due to internal one-point calibration, which might enhance reproducibility as it excludes any possible error caused by external calibration. The $\mathrm{RSD}_{\mathrm{r}}$ and $\mathrm{RSD}_{\mathrm{R}}$ values for 3-MCPD were between $1.5 \%$ and $15.6 \%$ and between $7.8 \%$ and $18.2 \%$, respectively. The $\mathrm{RSD}_{\mathrm{r}}$ results for 2 -MCPD were calculated to be $2.6 \%$ to $20.8 \%$ while the $\mathrm{RSD}_{\mathrm{R}}$ values showed to be $10.0 \%$ to $23.1 \%$. The study revealed a surprisingly high sensitivity for 3-MCPD and an even greater sensitivity for 2-MCPD. In fact, 2-MCPD occurs commonly in most refined oils and fats in amounts that are approximately one third 
of the corresponding 3-MCPD content. This ratio was also found in the food emulsifier samples included in this trial and consequently, 2-MCPD was always present in significantly smaller concentrations as 3-MCPD. At the same time, the lowest number of result outliers was related to 2-MCPD. Taking into account the lower 2-MCPD levels, the data can be interpreted as an indication that repeatability and reproducibility were slightly better than expected for this analyte in comparison to 3-MCPD. The reason for this could be varying background levels of 3-MCPD, a common issue due to possible 3-MCPD contamination of consumables such as laboratory glassware, plastic devices, packaging materials etc. In contrast, the same issue has rarely been reported for 2-MCPD. Background contamination might have a negative impact on the method performance, especially at these low concentration levels. However, no serious evaluation can be made based on the present results as no specific advice on how to deal with background levels was given in the method SOP. Another impact on the method performance was indicated by the comparably high $\mathrm{RSD}_{\mathrm{r}}$ and $\mathrm{RSD}_{\mathrm{R}}$ values for 3MCPD in "Olive Tree", which contained glycidol in an approximately eightyfold excess to 3-MCPD and in a two hundred and fiftyfold excess to 2-MCPD. The reason for this effect, which occurred more significantly with 3-MCPD than with 2-MCPD, might be the large amounts of glycidol present. Another possibility is that traces of chloride being present in the sample itself or existing as is a common impurity of the reagents or consumables used during sample preparation, might react with GE or the free glycidol released during interesterification to form small quantities of induced 3-MCPD. Naturally, this effect is hardly reproducible and would therefore impact the precision of the method.

\section{Conclusions}

A method for the determination of free and ester-bound 2- and 3-MCPD as well as ester-bound glycidol in glycerol-based food emulsifiers was successfully validated in a collaborative trial. The method that appears as a modification of AOCS Official Method Cd 29b-13 (ISO 18363-2) showed applicability for the determination of the aforementioned analytes in food emulsifiers classified as E 471, E 475 and E 476. The limits of quantification, expressed as method applicability are $0.22 \mathrm{mg} / \mathrm{kg}$ for glycidol being present as GE, 0.06 $\mathrm{mg} / \mathrm{kg}$ for total 3-MCPD and $0.02 \mathrm{mg} / \mathrm{kg}$ for total 2-MCPD.

\section{Acknowledgments}

We thank the following collaborators for their participation:

Helle Lochmann \& Annette Tjornelund Jensen, DuPont Nutrition Biosciences ApS, Brabrand, Denmark

Katja Kjelgaard Baumann \& Johnny Wittendorf Madsen, Dupont Nutrition \& Health, Grindsted, Denmark

Adrian Burke \& Julie Vichier, Kerry EMEA Global Technology \& Innovation Centre, Kildare, Ireland

Ramzi Sellik, Oleon Innovation, Compiegne Cedex, France

Mikkel Bach Skovsgaard \& Britta Japp, Palsgaard A/S, Juelsminde, Denmark

\section{References}


Abraham, K., Appel, K. E., Berger-Preiss, Apel, E., Gerling, S., Mielke, H., Creutzenberg, O. \& Lampen, A. (2013). Relative oral bioavailability of 3-MCPD from 3-MCPD fatty acid esters in rats. Arch Toxicol 87: 694-659.

Abraham, K. Hielscher, J., Kuhlmann, J. \& Monien, B. H. (2021). Urinary Excretion of 2/3-Monochloropropanediol (2/3-MCPD) and 2,3-Dihydroxypropylmercapturic Acid (DHPMA) after a Single High dose of Fatty Acid Esters of 2/3-MCPD and Glycidol: A Controlled Exposure Study in Humans. Mol Nutr Food Res 65 : 200735.

Appel, K. E., Abraham, K., Berger-Preiss, E., Hansen, T., Apel, E., Schuchardt, S. Vogt, C. Bakhiya, N., Creutzenberg, O. \& Lampen, A. (2013). Relative oral bioavailability of glycidol from glycidyl fatty acid esters in rats. Arch Toxicol 87: 1649-1659

AOCS Official Method Cd 29a-13. 2-and 3-MCPD Fatty Acid Esters and Glycidol Fatty Acid Esters in Edible Oils and Fats by Acid Transesterification and GC/MS. Revised 2017

AOCS Official Method Cd 29b-13. 2-and 3-MCPD Fatty Acid Esters and Glycidol Fatty Acid Esters in Edible Oils and Fats by Alkaline Transesterification and GC/MS. Revised 2017

AOCS Official Method Cd 29c-13. 2-and 3-MCPD Fatty Acid Esters and Glycidol Fatty Acid Esters in Edible Oils and Fats by GC/MS (Difference Method). Revised 2017

DGF (Deutsche Gesellschaft fuer Fettwissenschaft) (2011). DGF Standard Method C-VI 18 (10)

Fatty-acid-bound 3-chloropropane-1,2-diol (3-MCPD) and 2,3-epoxipropane-1-ol (glycidol).

Determination in oils and fats by GC/MS (Differential measurement). Deutsche Einheitsmethoden zur Untersuchung von Fetten, Fettprodukten, Tensiden und verwandten Stoffen.

EFSA CONTAM Panel (EFSA Panel on Contaminants in the Food Chain) (2016): Risks for human health related to the presence of 3- and 2-monochloropropanediol (MCPD), and their fatty acid esters, and glycidyl fatty acid esters in food. EFSA Journal 14(5): 4426.

EFSA ANS Panel (EFSA Panel on Food Additives and Nutrient Sources added to Food) (2017-a). Scientific opinion: re-evaluation of mono- and diglycerides of fatty acids (E 471) as food additives. EFSA Journal 15(11): 5045.

EFSA ANS Panel (EFSA Panel on Food Additives and Nutrient Sources added to Food) (2017-b). Scientific opinion: re-evaluation of polyglycerol esters of fatty acids (E 475) as a food additive. EFSA Journal 15(12), 5089.

EFSA ANS Panel (EFSA Panel on Food Additives and Nutrient Sources added to Food) (2017-c). Scientific opinion: re-evaluation of polyglycerol polyricinoleate (E 476) as a food additive. EFSA Journal 15(3): 4743.

EFSA (2018). Call for technical and toxicological data on mono- and di-glycerides of fatty acids (E 471) for uses as a food additive in foods for all population groups including infants below 16 weeks of age. Available at: http://www.efsa.europa.eu/en/consultations/call/call-technical-and-toxicological-datamono-and-di-glycerides-fatty

EC (2018-a). Call for technical data on the permitted food additive polyglycerol esters of fatty acids (E 475). Available at: fs_food-improvementagents_reeval_call_20181123_e475_data.pdf (europa.eu) EC (2018-b). Call for technical data on the permitted food additive polyglycerol polyricinoleate (E 476). Available at: fs_food-improvement-agents_reeval_call_20181123_e476_data.pdf (europa.eu) EU (2010). Commission Regulation (EU) No 257/2010 of 25 March 2010 setting up a programme for the re-evaluation of approved food additives in accordance with Regulation (EC) No 1333/2008 of the European Parliament and of the Council on food additives. OJEU, L8: 19-27

EU (2020). COMMISSION REGULATION (EU) 2020/1322 of 23 September 2020 amending Regulation (EC) No 1881/2006 as regards maximum levels of 3-monochloropropanediol (3-MCPD), 3-MCPD fatty acid esters and glycidyl fatty acid esters in certain foods. OJEU, L310. 
Abraham, K., Appel, K. E., Berger-Preiss, Apel, E., Gerling, S., Mielke, H., Creutzenberg, O. \& Lampen, A. (2013). Relative oral bioavailability of 3-MCPD from 3-MCPD fatty acid esters in rats. Arch Toxicol 87: 694-659.

Abraham, K. Hielscher, J., Kuhlmann, J. \& Monien, B. H. (2021). Urinary Excretion of 2/3-Monochloropropanediol (2/3-MCPD) and 2,3-Dihydroxypropylmercapturic Acid (DHPMA) after a Single High dose of Fatty Acid Esters of 2/3-MCPD and Glycidol: A Controlled Exposure Study in Humans. Mol Nutr Food Res 65 : 200735.

Appel, K. E., Abraham, K., Berger-Preiss, E., Hansen, T., Apel, E., Schuchardt, S. Vogt, C. Bakhiya, N., Creutzenberg, O. \& Lampen, A. (2013). Relative oral bioavailability of glycidol from glycidyl fatty acid esters in rats. Arch Toxicol 87: 1649-1659

AOCS Official Method Cd 29a-13. 2-and 3-MCPD Fatty Acid Esters and Glycidol Fatty Acid Esters in Edible Oils and Fats by Acid Transesterification and GC/MS. Revised 2017

Hamlet, C. G., Sadd, P. A., Crews, C., Velisek, J. \& Baxter, D. E. (2002). Occurrence of 3-chloro-propane-1,2-diol (3-MCPD) and related compounds in foods: a review. Food Addit Contam 19(7): 619-631. Horwitz, W. \& Albert, R. (2006). The Horwitz ratio (HorRat): A useful index of method performance with respect to precision. J AOAC Int 89(4): 1095-1109. International Agency for Research on Cancer (2000). IARC Monographs, 77: 469-486. International Agency for Research on Cancer (2012). IARC Monographs, 110: 349-374. ISO (International Organization for Standardization) (1994). ISO 5725-2: 1994. Accuracy (trueness and precision) of measurement methods and results Part 2: Basic method for the determination of repeatability and reproducibility of a standard measurement method.

ISO (International Organization for Standardization) (2015). ISO 18363-1:2015. Determination of fatty-acid-bound chloropropanediols (MCPDs) and glycidol by GC/MS; Part 1: Method using fast alkaline transesterification and measurement for 3-MCPD and differential measurement for glycidol. In: ISO Standards Catalogue. Geneva, Switzerland.

ISO (International Organization for Standardization) (2018). ISO 18363-2:2018. Determination of fatty-acid-bound chloropropanediols (MCPDs) and glycidol by GC/MS; Part 2: Method using slow alkaline transesterification and measurement for 2-MCPD, 3-MCPD and glycidol. In: ISO Standards Catalogue. Geneva, Switzerland.

ISO (International Organization for Standardization) (2017). ISO 18363-3:2017. Determination of fatty-acid-bound chloropropanediols (MCPDs) and glycidol by GC/MS; Part 3: Method using acid transesterification and measurement for 2-MCPD, 3-MCPD and glycidol. In: ISO Standards Catalogue. Geneva, Switzerland. Kuhlmann, J. (2011). Determination of bound 2,3-epoxy-1-propanol (glycidol) and bound monochloropropanediol (MCPD) in refined oils. Eur J Lipid Sci Technol 113: 335-344. Weisshaar, R., Perz, R. (2010). Fatty acid esters of glycidol in refined fats and oils. Eur J Lipid Sci Technol 112: 158-165. Zelinkova, Z., Svejkovska B., Velisek J. \& Dolezal M. (2006). Fatty esters of 3-chloropropane-1,2-diol in edible oils. Food Addit Contam 23(12): 1290-1298.

Zelinkova, Z., Giri, A. \& Wenzl, T. (2017). Assessment of critical steps of a GC/MS based indirect analytical method for the determination of fatty acid esters of monochloropropanediols (MCPDEs) and of glycidol (GEs). Food Control 77: 65-75.

\section{Table 1: Description of samples.}

Sample classification, composition

(physical state)

E 471, DMG SAFA (powder)

E 471, DMG SAFA (powder)
Anonymised label

Ink

Scissors
Approximate analyte levels from pre-testing

No or minor analyte contents

Glycidol $<<1 \mathrm{mg} / \mathrm{kg}$,

(2-)3-MCPDE $<2.5 \mathrm{mg} / \mathrm{kg}$ 


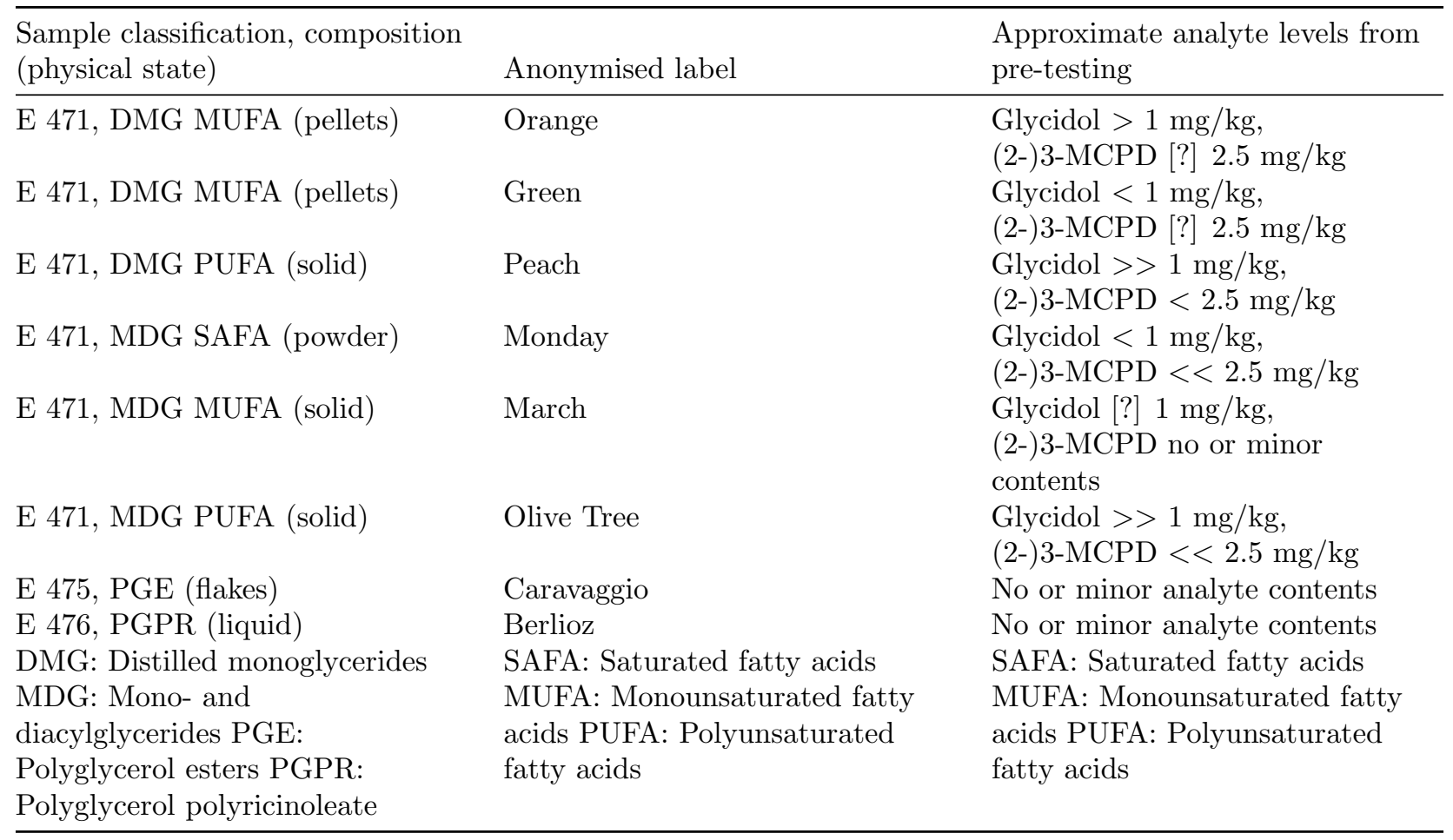

Table 2.1: Validation summary for E471 distilled monoglycerides.

E 471 distilled monoglycerides

Ink: E 471, DMG SAFA

Valid data sets $[\mathrm{n}]$

Mean $[\mathrm{mg} / \mathrm{kg}]$

Repeatability* $\mathrm{RSD}_{\mathrm{r}}[\%]$

Reproducibility* $\mathrm{RSD}_{\mathrm{R}}[\%]$

Reproducibility limit $[\mathrm{mg} / \mathrm{kg}]$

HorRat $\mathrm{R}$ value

Mean value quantifiable

Scissors: E 471, DMG SAFA

Valid data sets $[\mathrm{n}]$

Mean $[\mathrm{mg} / \mathrm{kg}]$

Repeatability* $\mathrm{RSD}_{\mathrm{r}}[\%]$

Reproducibility* RSD ${ }_{\mathrm{R}}[\%]$

Reproducibility limit $[\mathrm{mg} / \mathrm{kg}]$

HorRat $R$ value

Mean value quantifiable

Orange: E 471, DMG MUFA

Valid data sets [n]

Mean $[\mathrm{mg} / \mathrm{kg}]$

Repeatability* $\mathrm{RSD}_{\mathrm{r}}$ [\%]

Reproducibility* $\mathrm{RSD}_{\mathrm{R}}$ [\%]

Reproducibility limit $[\mathrm{mg} / \mathrm{kg}]$ 
HorRat $\mathrm{R}$ value

Mean value quantifiable

Green: E 471, DMG PUFA

Valid data sets $[\mathrm{n}]$

Mean $[\mathrm{mg} / \mathrm{kg}]$

Repeatability* $\mathrm{RSD}_{\mathrm{r}}$ [\%]

Reproducibility* $\operatorname{RSD}_{\mathrm{R}}[\%]$

Reproducibility limit $[\mathrm{mg} / \mathrm{kg}]$

HorRat $\mathrm{R}$ value

Mean value quantifiable

Peach: E 471, DMG PUFA

Valid data sets [n]

Mean $[\mathrm{mg} / \mathrm{kg}]$

Repeatability* $\mathrm{RSD}_{\mathrm{r}}$ [\%]

Reproducibility* $\mathrm{RSD}_{\mathrm{R}}[\%]$

Reproducibility limit $[\mathrm{mg} / \mathrm{kg}]$

HorRat $R$ value

Mean value quantifiable

: Relative Standard Deviation ${ }^{1)}$ glycidyl fatty acid esters expressed as glycidol ${ }^{2)}$ sum of free MCPD and MCPD fatty acid

Table 2.2: Validation summary for E471 mono- and diglycerides.

\section{E 471 mono- and diglycerides}

Monday: E 471, MDG SAFA

Valid data sets [n]

Mean $[\mathrm{mg} / \mathrm{kg}]$

Repeatability* $\mathrm{RSD}_{\mathrm{r}}$ [\%]

Reproducibility* $\mathrm{RSD}_{\mathrm{R}}[\%]$

Reproducibility limit $[\mathrm{mg} / \mathrm{kg}]$

HorRat $\mathrm{R}$ value

Mean value quantifiable

March: E 471, MDG MUFA

Valid data sets [n]

Mean $[\mathrm{mg} / \mathrm{kg}]$

Repeatability* $\mathrm{RSD}_{\mathrm{r}}[\%]$

Reproducibility* $\mathrm{RSD}_{\mathrm{R}}[\%]$

Reproducibility limit $[\mathrm{mg} / \mathrm{kg}]$

HorRat $\mathrm{R}$ value

Mean value quantifiable

Olive Tree: E 471, MDG PUFA

Valid data sets [n]

Mean $[\mathrm{mg} / \mathrm{kg}]$

Repeatability* RSDr [\%]

Reproducibility* RSD $_{\mathrm{R}}$ [\%]

Reproducibility limit $[\mathrm{mg} / \mathrm{kg}]$

HorRat $\mathrm{R}$ value

Mean value quantifiable

: Relative Standard Deviation ${ }^{1)}$ glycidyl fatty acid esters expressed as glycidol ${ }^{2)}$ sum of free MCPD and MCPD fatty acid 
Table 2.3 : Validation summary for E 475 and E 476 polyglycerol esters.

E 475, E 476 polyglycerol esters

Caravaggio: E 475, PGE

Valid data sets $[\mathrm{n}]$

Mean $[\mathrm{mg} / \mathrm{kg}]$

Repeatability* $\mathrm{RSD}_{\mathrm{r}}[\%]$

Reproducibility* $\mathrm{RSD}_{\mathrm{R}}[\%]$

Reproducibility limit $[\mathrm{mg} / \mathrm{kg}]$

HorRat $\mathrm{R}$ value

Mean value quantifiable

Berlioz: E 476, PGPR

Valid data sets [n]

Mean $[\mathrm{mg} / \mathrm{kg}]$

Repeatability* $\mathrm{RSD}_{\mathrm{r}}[\%]$

Reproducibility* $\operatorname{RSD}_{\mathrm{R}}[\%]$

Reproducibility limit $[\mathrm{mg} / \mathrm{kg}]$

HorRat $R$ value

Mean value quantifiable

: Relative Standard Deviation ${ }^{1)}$ glycidyl fatty acid esters expressed as glycidol ${ }^{2)}$ sum of free MCPD and MCPD fatty acid

Table 3: Summary validation results for $\mathrm{RSD}_{\mathbf{r}}, \mathrm{RSD}_{\mathrm{R}}$ and HorRat $\mathrm{R}$ values.

\section{glycidol ${ }^{1)}$}

3-MCPD ${ }^{2)}$

2-MCPD 2)

1) glycidyl fatty acid esters expressed as glycidol ${ }^{2)}$ sum of free MCPD and MCPD fatty acid esters expressed as MCPD 